\title{
BOUNDS FOR ELLIPTIC OPERATORS IN WEIGHTED SPACES
}

\author{
LOREDANA CASO
}

Received 24 November 2004; Accepted 28 September 2005

Some estimates for solutions of the Dirichlet problem for second-order elliptic equations are obtained in this paper. Here the leading coefficients are locally VMO functions, while the hypotheses on the other coefficients and the boundary conditions involve a suitable weight function.

Copyright (c) 2006 Loredana Caso. This is an open access article distributed under the Creative Commons Attribution License, which permits unrestricted use, distribution, and reproduction in any medium, provided the original work is properly cited.

\section{Introduction}

Let $\Omega$ be a bounded open subset of $\mathbb{R}^{n}, n \geq 3$, and let

$$
L=\sum_{i, j=1}^{n} a_{i j}(x) \frac{\partial^{2}}{\partial x_{i} \partial x_{j}}+\sum_{i=1}^{n} a_{i}(x) \frac{\partial}{\partial x_{i}}+a(x)
$$

be a uniformly elliptic operator with measurable coefficients in $\Omega$. Several bounds for the solutions of the problem

$$
\begin{gathered}
L u \geq f, \quad f \in L^{p}(\Omega), \\
u \in W^{2, p}(\Omega) \cap C^{o}(\bar{\Omega}), \\
u_{\mid \partial \Omega} \leq 0,
\end{gathered}
$$

$(p \in] n / 2,+\infty[$ ) have been given, and the application of such estimates allows to obtain certain uniqueness results for $(D)$.

For instance, if $p \geq n, a_{i}, a \in L^{p}(\Omega)$ (with $a \leq 0$ ), a classical result of Pucci [4] shows that any solution $u$ of the problem $(D)$ verifies the bound

$$
\sup _{\Omega} u \leq K\|f\|_{L^{p}(\Omega)}
$$

where $K \in \mathbb{R}_{+}$depends on $\Omega, n, p,\left\|a_{i}\right\|_{L^{p}(\Omega)}$ and on the ellipticity constant. 
The case $p<n$, where additional hypotheses on the leading coefficients are necessary, has been studied by several authors. Recently, a uniqueness result has been obtained in [3] under the assumption that the $a_{i j}$ 's are of class VMO, $a_{i}=a=0$ and $\left.p \in\right] 1,+\infty$ [. This theorem has been extended to the case $a_{i} \neq 0, a \neq 0$ in [7].

If $\Omega$ is an arbitrary open subset of $\mathbb{R}^{n}$ and $\left.p \in\right] n / 2,+\infty$ [, a bound of type (1.2) and a consequent uniqueness result can be found in [1]. In fact, it has been proved there that if the coefficients $a_{i j}$ are bounded and locally VMO, the coefficients $a_{i}$, a satisfy suitable

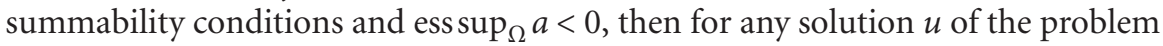

$$
\begin{gathered}
L u \geq f, \quad f \in L_{\mathrm{loc}}^{p}(\Omega), \\
u \in W_{\mathrm{loc}}^{2, p}(\Omega) \cap C^{o}(\bar{\Omega}), \\
u_{\mid \partial \Omega} \leq 0, \\
\limsup _{|x| \rightarrow+\infty} u(x) \leq 0 \quad \text { if } \Omega \text { is unbounded, }
\end{gathered}
$$

there exist a ball $B \subset \subset \Omega$ and a constant $c \in \mathbb{R}_{+}$such that

$$
\sup _{\Omega} u \leq c\left(f_{B}\left|f^{-}\right|^{p} d x\right)^{1 / p},
$$

where $f^{-}$is the negative part of $f$,

$$
f_{B}\left|f^{-}\right|^{p} d x=\frac{1}{|B|} \int_{B}\left|f^{-}\right|^{p} d x
$$

and $c$ depends on $n$, $p$, on the ellipticity constant, and on the regularity of the coefficients of $L$.

The aim of this paper is to study a problem similar to that considered in [1], but with boundary conditions depending on an appropriate weight function. More precisely, fix a weight function $\sigma \in \mathscr{A}(\Omega) \cap C^{\infty}(\Omega)$ (see Section 2 for the definition of $\mathscr{A}(\Omega)$ ) and $s \in \mathbb{R}$, we consider a solution $u$ of the problem

$$
\begin{gathered}
L u \geq f, \quad f \in L_{\mathrm{loc}}^{p}(\Omega), \\
u \in W_{\mathrm{loc}}^{2, p}(\Omega), \\
\limsup _{x \rightarrow x_{o}} \sigma^{s}(x) u(x) \leq 0 \quad \forall x_{o} \in \partial \Omega, \\
\limsup _{|x| \rightarrow+\infty}^{s}(x) u(x) \leq 0 \quad \text { if } \Omega \text { is unbounded. }
\end{gathered}
$$

If the coefficients $a_{i j}$ are bounded and locally VMO, the functions $\sigma a_{i}$ and $\sigma^{2} a$ are bounded and $\operatorname{essup}_{\Omega} \sigma^{2} a<0$, we will prove that there exist a ball $B \subset \subset \Omega$ and a constant $c_{o} \in \mathbb{R}_{+}$such that

$$
\sup _{\Omega} \sigma^{s} u \leq c_{o}\left(f_{B}\left|\sigma^{s+2} f^{-}\right|^{p} d x\right)^{1 / p},
$$

where $c_{o}$ depends on $n, p, s, \sigma$, on the ellipticity constant, and on the regularity of the coefficients of $L$. As a consequence, some uniqueness results are also obtained. 


\section{Notation and function spaces}

Let $\Omega$ be an open subset of $\mathbb{R}^{n}$ and let $\Sigma(\Omega)$ be the collection of all Lebesgue measurable subsets of $\Omega$. For each $E \in \Sigma(\Omega)$, we denote by $|E|$ the Lebesgue measure of $E$ and put

$$
E(x, r)=E \cap B(x, r) \quad \forall x \in \mathbb{R}^{n}, \forall r \in \mathbb{R}_{+},
$$

where $B(x, r)$ is the open ball in $\mathbb{R}^{n}$ of radius $r$ centered at $x$.

Denote by $\mathscr{A}(\Omega)$ the class of measurable functions $\rho: \Omega \rightarrow \mathbb{R}_{+}$such that

$$
\beta^{-1} \rho(y) \leq \rho(x) \leq \beta \rho(y) \quad \forall y \in \Omega, \forall x \in \Omega(y, \rho(y)),
$$

where $\beta \in \mathbb{R}_{+}$is independent of $x$ and $y$. For $\rho \in \mathscr{A}(\Omega)$, we put

$$
S_{\rho}=\left\{z \in \partial \Omega: \lim _{x \rightarrow z} \rho(x)=0\right\} .
$$

It is known that

$$
\rho \in L_{\mathrm{loc}}^{\infty}(\bar{\Omega}), \quad \rho^{-1} \in L_{\mathrm{loc}}^{\infty}\left(\bar{\Omega} \backslash S_{\rho}\right),
$$

and, if $S_{\rho} \neq \varnothing$,

$$
\rho(x) \leq \operatorname{dist}\left(x, S_{\rho}\right) \quad \forall x \in \Omega
$$

(see $[2,6]$ ). Having fixed $\rho \in \mathscr{A}(\Omega)$ such that $S_{\rho}=\partial \Omega$, it is possible to find a function $\sigma \in \mathscr{A}(\Omega) \cap C^{\infty}(\Omega) \cap C^{0,1}(\bar{\Omega})$ which is equivalent to $\rho$ and such that

$$
\begin{gathered}
\sigma \in L_{\mathrm{loc}}^{\infty}(\bar{\Omega}), \quad \sigma^{-1} \in L_{\mathrm{loc}}^{\infty}(\Omega), \\
\sigma(x) \leq \operatorname{dist}(x, \partial \Omega) \quad \forall x \in \Omega, \\
\left|\partial^{\alpha} \sigma(x)\right| \leq c_{\alpha} \sigma^{1-|\alpha|}(x) \quad \forall x \in \Omega, \forall \alpha \in \mathbb{N}_{o}^{n}, \\
\gamma^{-1} \sigma(y) \leq \sigma(x) \leq \gamma \sigma(y) \quad \forall y \in \Omega, \forall x \in \Omega(y, \sigma(y)),
\end{gathered}
$$

where $c_{\alpha}, \gamma \in \mathbb{R}_{+}$are independent of $x$ and $y$ (see [6]). For more properties of functions of $\mathscr{A}(\Omega)$ we refer to $[2,6]$.

If $\Omega$ has the property

$$
\left.\left.|\Omega(x, r)| \geq A r^{n} \quad \forall x \in \Omega, \forall r \in\right] 0,1\right],
$$

where $A$ is a positive constant independent of $x$ and $r$, it is possible to consider the space $\operatorname{BMO}(\Omega, t), t \in \mathbb{R}_{+}$, of functions $g \in L_{\text {loc }}^{1}(\bar{\Omega})$ such that

$$
[g]_{\mathrm{BMO}(\Omega, t)}=\sup _{\substack{x \in \Omega \\ r \in[0, t]}} f_{\Omega(x, r)}\left|g-f_{\Omega(x, r)} g\right| d y<+\infty,
$$

where $f_{\Omega(x, r)} g d y=1 /|\Omega(x, r)| \int_{\Omega(x, r)} g d y$. If $g \in \operatorname{BMO}(\Omega)=\operatorname{BMO}\left(\Omega, t_{A}\right)$, where

$$
t_{A}=\sup \left\{t \in \mathbb{R}_{+}: \sup _{\substack{x \in \Omega \\ r \in[0, t]}} \frac{r^{n}}{|\Omega(x, r)|} \leq \frac{1}{A}\right\},
$$


4 Bounds for elliptic operators in weighted spaces

we will say that $g \in \operatorname{VMO}(\Omega)$ if $[g]_{\mathrm{BMO}(\Omega, t)} \rightarrow 0$ for $t \rightarrow 0^{+}$. A function $\eta[g]: \mathbb{R}_{+} \rightarrow \mathbb{R}_{+}$is called a modulus of continuity of $g$ in $\operatorname{VMO}(\Omega)$ if

$$
\begin{gathered}
\mathrm{BMO}(\Omega, t) \leq \eta[g](t) \quad \forall t \in \mathbb{R}_{+}, \\
\lim _{t \rightarrow 0^{+}} \eta[g](t)=0 .
\end{gathered}
$$

We say that $g \in \mathrm{VMO}_{\text {loc }}(\Omega)$ if $(\zeta g)_{o} \in \operatorname{VMO}\left(\mathbb{R}^{n}\right)$ for any $\zeta \in C_{o}^{\infty}(\Omega)$, where $(\zeta g)_{o}$ denotes the zero extension of $\zeta g$ outside of $\Omega$. A more detailed account of properties of the above defined spaces $\operatorname{BMO}(\Omega)$ and $\operatorname{VMO}(\Omega)$ can be found in [5].

\section{An a priori bound}

Fix $p \in] n / 2,+\infty\left[\right.$. Let $B$ be an open ball of $\mathbb{R}^{n}, n \geq 3$, of radius $\delta$. We consider in $B$ the differential operator

$$
L_{B}=\sum_{i, j=1}^{n} \alpha_{i j}(x) \frac{\partial^{2}}{\partial x_{i} \partial x_{j}}+\sum_{i=1}^{n} \alpha_{i}(x) \frac{\partial}{\partial x_{i}}+\alpha(x)
$$

with the following condition on the coefficients:

$$
\begin{gathered}
\alpha_{i j}=\alpha_{j i} \in L^{\infty}(B) \cap \operatorname{VMO}(B), \quad i, j=1, \ldots, n, \\
\exists \mu \in \mathbb{R}_{+}: \sum_{i, j=1}^{n} \alpha_{i j} \zeta_{i} \zeta_{j} \geq \mu|\zeta|^{2} \quad \text { a.e. in } B, \forall \zeta \in \mathbb{R}^{n}, \\
\alpha_{i} \in L^{\infty}(B), \quad i=1, \ldots, n, \alpha \in L^{\infty}(B), \alpha \leq 0 \text { a.e. in } B .
\end{gathered}
$$

Let $\mu_{0}, \mu_{1}, \mu_{2} \in \mathbb{R}_{+}$such that

$$
\sum_{i, j=1}^{n}\left\|\alpha_{i j}\right\|_{L^{\infty}(B)} \leq \mu_{0}, \quad \delta \sum_{1=1}^{n}\left\|\alpha_{i}\right\|_{L^{\infty}(B)} \leq \mu_{1}, \quad \delta^{2}\|\alpha\|_{L^{\infty}(B)} \leq \mu_{2} .
$$

Note that under the assumption $\left(h_{B}\right)$, the operator $L_{B}$ from $W^{2, p}(B)$ into $L^{p}(B)$ is bounded and the estimate

$$
\left\|L_{B} u\right\|_{L^{p}(B)} \leq c_{1}\|u\|_{W^{2, p}(B)} \quad \forall u \in W^{2, p}(B)
$$

holds, where $c_{1} \in \mathbb{R}_{+}$depends on $n, p, \mu_{0}, \mu_{1}, \mu_{2}$.

Lemma 3.1. Suppose that condition $\left(h_{B}\right)$ is verified, and let $u$ be a solution of the problem

$$
\begin{gathered}
u \in W^{2, p}(B), \\
L_{B} u \geq \phi, \quad \phi \in L^{p}(B), \\
u_{\left.\right|_{\partial B}} \leq 0 .
\end{gathered}
$$

Then there exists $c \in \mathbb{R}_{+}$such that

$$
\sup _{B} u \leq c \delta^{2-n / p}\left\|\phi^{-}\right\|_{L^{p}(B)},
$$


where $c$ depends on $n, p, \mu, \mu_{0}, \mu_{1}, \mu_{2},\left[p\left(\alpha_{i j}\right)\right]_{\mathrm{BMO}\left(R^{n}, \cdot\right)}$, and where $p\left(\alpha_{i j}\right)$ is an extension of $\alpha_{i j}$ to $\mathbb{R}^{n}$ in $L^{\infty}\left(\mathbb{R}^{n}\right) \cap \operatorname{VMO}\left(\mathbb{R}^{n}\right)$.

Proof. Put $B=B(y, \delta)$, where $y$ is the centre of $B$, and $B^{*}=B(y, 1)$.

Consider the function $T: B \rightarrow B^{*}$ defined by the position

$$
T(x)=y+\frac{x-y}{\delta}=z,
$$

and for each function $g$ defined on $B$, put $g^{*}=g \circ T^{-1}$.

We observe that

$$
L_{B}^{*} u^{*}=\delta^{2}\left(L_{B} u\right)^{*}
$$

where

$$
L_{B}^{*}=\sum_{i, j=1}^{n} \alpha_{i j}^{*}(z) \frac{\partial^{2}}{\partial z_{i} \partial z_{j}}+\delta \sum_{i=1}^{n} \alpha_{i}^{*}(z) \frac{\partial}{\partial z_{i}}+\delta^{2} \alpha^{*}(z) .
$$

Denote by $p\left(\alpha_{i j}\right)$ an extension of $\alpha_{i j}$ to $\mathbb{R}^{n}$ such that

$$
p\left(\alpha_{i j}\right) \in L^{\infty}\left(\mathbb{R}^{n}\right) \cap \operatorname{VMO}\left(\mathbb{R}^{n}\right)
$$

(for the existence of such function see [5, Theorem 5.1]). Since

$$
p\left(\alpha_{i j}\right)^{*} \in L^{\infty}\left(\mathbb{R}^{n}\right) \cap \operatorname{VMO}\left(\mathbb{R}^{n}\right), \quad p\left(\alpha_{i j}\right)_{\left.\right|_{B^{*}}}^{*}=\alpha_{i j}^{*}
$$

it follows that

$$
\alpha_{i j}^{*} \in L^{\infty}\left(B^{*}\right) \cap \operatorname{VMO}\left(B^{*}\right)
$$

Moreover, the condition $\left(h_{B}\right)$ yields that

$$
\begin{gathered}
\alpha_{i j}^{*}=\alpha_{j i}^{*}, \quad i, j=1, \ldots, n, \\
\sum_{i, j=1}^{n} \alpha_{i j}^{*} \zeta_{i} \zeta_{j} \geq \mu|\zeta|^{2} \quad \text { a.e. in } B^{*}, \forall \zeta \in \mathbb{R}^{n}, \\
\alpha_{i}^{*} \in L^{\infty}\left(B^{*}\right), \quad i=1, \ldots, n, \quad \alpha^{*} \in L^{\infty}\left(B^{*}\right), \quad \alpha^{*} \leq 0 \quad \text { a.e. in } B^{*} .
\end{gathered}
$$

We observe that the condition (3.12) implies that for $r, s \in] 1,+\infty$ [ the modulus of continuity of $\delta \alpha_{i}^{*}$ in $L^{r}\left(B^{*}\right)$ and that of $\delta^{2} \alpha^{*}$ in $L^{s}\left(B^{*}\right)$ depend only on $\left\|\delta \alpha_{i}^{*}\right\|_{L^{\infty}\left(B^{*}\right)}$ and $\left\|\delta^{2} \alpha^{*}\right\|_{L^{\infty}\left(B^{*}\right)}$, respectively.

Thus, applying (3.10), (3.12), and [7, Theorem 2.1], it follows that the problem

$$
\begin{gathered}
L_{B}^{*} v=\psi \in L^{p}\left(B^{*}\right), \\
v \in \mathrm{W}^{2, p}\left(B^{*}\right) \cap \stackrel{o}{W^{1, p}}\left(B^{*}\right)
\end{gathered}
$$


6 Bounds for elliptic operators in weighted spaces

has a unique solution $v$ satisfying the estimate

$$
\|v\|_{W^{2, p\left(B^{*}\right)}} \leq K\|\psi\|_{L^{p}\left(B^{*}\right)},
$$

where $K$ depends on $n, p, \mu, \mu_{0}, \mu_{1}, \mu_{2},\left[p\left(\alpha_{i j}\right)^{*}\right]_{\mathrm{BMO}\left(R^{n}, \cdot\right) \cdot}$.

The estimate (3.5) follows now from (3.14) using the same arguments of the proof of Lemma 3.2 [1] in order to obtain there $\left(e_{B}\right)$ from $[1,(3.23)]$.

\section{Hypotheses and preliminary results}

Let $\Omega$ be an open subset of $\mathbb{R}^{n}, n \geq 3$. Fix $\rho \in \mathscr{A}(\Omega) \cap L^{\infty}(\Omega)$ such that $S_{\rho}=\partial \Omega$.

Consider a function $g \in C_{o}^{\infty}\left(\overline{\mathbb{R}}_{+}\right)$satisfying the condition

$$
0 \leq g \leq 1, \quad g(t)=1 \quad \text { if } t \geq 1, \quad g(t)=0 \quad \text { if } t \leq \frac{1}{2} .
$$

For any $k \in \mathbb{N}$, we put

$$
\eta_{k}(x)=\frac{1}{k} \zeta_{k}(x)+\left(1-\zeta_{k}(x)\right) \sigma(x), \quad x \in \Omega
$$

where $\zeta_{k}(x)=g(k \sigma(x)), x \in \Omega$. Clearly, $\eta_{k} \in C^{\infty}(\Omega)$ for any $k \in \mathbb{N}$ and

$$
\eta_{k}(x)= \begin{cases}\frac{1}{k} & \text { if } x \in \bar{\Omega}_{k}, \\ \sigma(x) & \text { if } x \in \Omega \backslash \Omega_{2 k},\end{cases}
$$

where

$$
\Omega_{k}=\left\{x \in \Omega: \sigma(x)>\frac{1}{k}\right\}, \quad k \in \mathbb{N} .
$$

In the following we will use the notation

$$
f_{x}=\left(\sum_{i=1}^{n} f_{x_{i}}^{2}\right)^{1 / 2}, \quad f_{x x}=\left(\sum_{i, j=1}^{n} f_{x_{i} x_{j}}^{2}\right)^{1 / 2}
$$

It is easy to show that for each $k \in \mathbb{N}$,

$$
\begin{gathered}
\sigma(x) \leq \eta_{k}(x) \leq 2 \sigma(x), \quad x \in \Omega \backslash \bar{\Omega}_{k}, \\
c_{k}^{\prime} \sigma(x) \leq \eta_{k}(x) \leq \sigma(x), \quad x \in \Omega_{k}, \\
\left(\eta_{k}(x)\right)_{x} \leq c_{1}(\sigma(x))_{x}, \quad x \in \Omega, \\
\left(\eta_{k}(x)\right)_{x x} \leq c_{2} \frac{(\sigma(x))_{x}^{2}+\sigma(x)(\sigma(x))_{x x}}{\sigma(x)}, \quad x \in \Omega,
\end{gathered}
$$


where $c_{k}^{\prime} \in \mathbb{R}_{+}$depends on $k$ and $\sigma$, and $c_{1}, c_{2} \in \mathbb{R}_{+}$depend only on $n$. Moreover, for any $s \in \mathbb{R}$, we have

$$
\begin{gathered}
\frac{\left(\eta_{k}^{s}(x)\right)_{x}}{\eta_{k}^{s}(x)} \leq c_{3} \frac{\left(\eta_{k}(x)\right)_{x}}{\sigma(x)}, \quad x \in \Omega, \\
\frac{\left(\eta_{k}^{s}(x)\right)_{x x}}{\eta_{k}^{s}(x)} \leq c_{3} \frac{\left(\eta_{k}(x)\right)_{x}^{2}+\eta_{k}(x)\left(\eta_{k}(x)\right)_{x x}}{\sigma^{2}(x)}, \quad x \in \Omega,
\end{gathered}
$$

where $c_{3} \in \mathbb{R}_{+}$depends on $s$ and $n$.

We consider in $\Omega$ the differential operator

$$
L=\sum_{i, j=1}^{n} a_{i j}(x) \frac{\partial^{2}}{\partial x_{i} \partial x_{j}}+\sum_{i=1}^{n} a_{i}(x) \frac{\partial}{\partial x_{i}}+a(x),
$$

and put

$$
L_{o}=\sum_{i, j=1}^{n} a_{i j}(x) \frac{\partial^{2}}{\partial x_{i} \partial x_{j}} .
$$

We will make the following assumption on the coefficients of $L$ :

$$
\begin{gathered}
a_{i j}=a_{j i} \in L^{\infty}(\Omega) \cap \operatorname{VMO}_{\mathrm{loc}}(\Omega), \quad i, j=1, \ldots, n, \\
\exists v, \nu_{0} \in \mathbb{R}_{+}: \sum_{i, j=1}^{n}\left\|a_{i j}\right\|_{L^{\infty}(\Omega)} \leq \nu_{0}, \quad \sum_{i, j=1}^{n} a_{i j} \zeta_{i} \zeta_{j} \geq v|\zeta|^{2} \quad \text { a.e. in } \Omega, \forall \zeta \in \mathbb{R}^{n}, \\
\exists \nu_{1}, \nu_{2} \in \mathbb{R}_{+}: \underset{\Omega}{\operatorname{ess} \sup }\left(\sigma(x) \sum_{i=1}^{n}\left|a_{i}(x)\right|\right) \leq \nu_{1}, \quad \underset{\Omega}{\operatorname{esssup}}\left(\sigma^{2}(x)|a(x)|\right) \leq \nu_{2}, \\
\exists a_{o} \in \mathbb{R}_{+}: \operatorname{ess\operatorname {sup}}\left(\sigma^{2}(x) a(x)\right)=-a_{o} .
\end{gathered}
$$

Fixed $s \in \mathbb{R}$, let $u$ be a solution of the problem

$$
\begin{aligned}
& L u \geq f, \quad f \in L_{\mathrm{loc}}^{p}(\Omega), \quad u \in W_{\mathrm{loc}}^{2, p}(\Omega), \\
& \limsup _{x \rightarrow x_{0}} \sigma^{s}(x) u(x) \leq 0 \quad \forall x_{o} \in \partial \Omega, \\
& \limsup _{|x| \rightarrow+\infty} \sigma^{s}(x) u(x) \leq 0 \quad \text { if } \Omega \text { is unbounded. }
\end{aligned}
$$

For any $k \in \mathbb{N}$, we put

$$
w_{k}(x)=\eta_{k}^{s}(x) u(x), \quad x \in \Omega \text {. }
$$


8 Bounds for elliptic operators in weighted spaces

Lemma 4.1. Suppose that condition $\left(h_{1}\right)$ holds. Then, for any $k \in \mathbb{N}$ there exist functions $b_{i}^{k}(i=1, \ldots, n), b^{k}, g^{k}$ and positive constants $\beta_{1}$ and $\beta_{2}$ such that

$$
\begin{gathered}
\underset{\Omega}{\operatorname{esssup}}\left(\sigma(x) \sum_{i=1}^{n}\left|b_{i}^{k}(x)\right|\right) \leq \beta_{1}, \\
\underset{\Omega}{\operatorname{ess} \sup }\left(\sigma^{2}(x)\left|b^{k}(x)\right|\right) \leq \beta_{2}, \\
g^{k} \in L_{\mathrm{loc}}^{p}(\Omega),
\end{gathered}
$$

where $\beta_{1}$ depends on $s, n, v_{0}, \nu_{1}$ and $\beta_{2}$ depends on $s, n, v_{0}, v_{2}$. Moreover, the function $w_{k}, k \in \mathbb{N}$, satisfies the following conditions:

$$
\begin{gathered}
w_{k} \in W_{\text {loc }}^{2, p}(\Omega), \quad \limsup _{x \rightarrow x_{o}} w_{k}(x) \leq 0 \quad \forall x_{o} \in \partial \Omega, \\
\limsup _{|x| \rightarrow+\infty} w_{k}(x) \leq 0 \quad \text { if } \Omega \text { is unbounded, } \\
L_{o} w_{k}+\sum_{i=1}^{n} b_{i}^{k}\left(w_{k}\right)_{x_{i}}+b^{k} w_{k} \geq g^{k} \quad \text { in } \Omega .
\end{gathered}
$$

Proof. Fix $k \in \mathbb{N}$. From (4.6)-(4.11) and from (2.6), (2.8), it easily follows that the function $w_{k}$, defined by (4.14), verifies (4.18).

Moreover, observe that

$$
\begin{gathered}
L_{o} w_{k}-u L_{o} \eta_{k}^{s}-2 \sum_{i, j=1}^{n} a_{i j}\left(\eta_{k}^{s}\right)_{x_{j}} u_{x_{i}}+\sum_{i=1}^{n} a_{i}\left(\eta_{k}^{s} u\right)_{x_{i}} \\
-u \sum_{i=1}^{n} a_{i}\left(\eta_{k}^{s}\right)_{x_{i}}+a \eta_{k}^{s} u=\eta_{k}^{s} L u, \quad x \in \Omega .
\end{gathered}
$$

Since

$$
\left(\eta_{k}^{s}\right)_{x_{j}} u_{x_{i}}=\left(\eta_{k}^{s} u\right)_{x_{i}} \frac{\left(\eta_{k}^{s}\right)_{x_{j}}}{\eta_{k}^{s}}-\frac{\left(\eta_{k}^{s}\right)_{x_{i}}\left(\eta_{k}^{s}\right)_{x_{j}}}{\left(\eta_{k}^{s}\right)^{2}}\left(\eta_{k}^{s} u\right),
$$

from (4.20), (4.19) follows, where we have put

$$
\begin{aligned}
b_{i}^{k} & =a_{i}-2 \sum_{j=1}^{n} a_{i j} \frac{\left(\eta_{k}^{s}\right)_{x_{j}}}{\eta_{k}^{s}}, \quad i=1, \ldots, n, \\
b^{k} & =a+2 \sum_{i, j=1}^{n} a_{i j} \frac{\left(\eta_{k}^{s}\right)_{x_{i}}\left(\eta_{k}^{s}\right)_{x_{j}}}{\left(\eta_{k}^{s}\right)^{2}}-\sum_{i, j=1}^{n} a_{i j} \frac{\left(\eta_{k}^{s}\right)_{x_{i} x_{j}}}{\eta_{k}^{s}} \\
g^{k} & =\eta_{k}^{s} f+\sum_{i=1}^{n} a_{i} \frac{\left(\eta_{k}^{s}\right)_{x_{i}}}{\eta_{k}^{s}} w_{k} .
\end{aligned}
$$

On the other hand, using the hypothesis $\left(h_{1}\right),(4.6)-(4.11)$, and (2.8) it is easy to show that there exist $\beta_{1} \in \mathbb{R}_{+}$depending on $s, n, v_{0}, v_{1}$ and $\beta_{2} \in \mathbb{R}_{+}$depending on $s, n, v_{0}, v_{2}$, such that (4.15), (4.16), (4.17) hold. 
Now we suppose that the following hypothesis on $\rho$ holds:

$$
\lim _{k \rightarrow+\infty}\left(\sup _{\Omega \backslash \Omega_{k}}\left((\sigma(x))_{x}+\sigma(x)(\sigma(x))_{x x}\right)\right)=0 .
$$

An example of function $\rho$ such that $\sigma$ satisfies $\left(h_{2}\right)$ is provided in [2].

Lemma 4.2. Suppose that conditions $\left(h_{1}\right)$ and $\left(h_{2}\right)$ hold. Then there exists $k_{o} \in \mathbb{N}$ such that

$$
\begin{gathered}
\underset{\Omega}{\operatorname{ess} \sup }\left(\sigma(x) \sum_{i=1}^{n}\left|b_{i}^{k_{o}}(x)\right|\right) \leq \nu_{1}+\frac{a_{o}}{2}, \\
\quad \underset{\Omega}{\operatorname{sssup}\left(\sigma^{2}(x) b^{k_{o}}(x)\right) \leq-\frac{a_{o}}{2},} \\
g^{k_{o}}(x) \geq \eta_{k_{o}}^{s}(x) f(x)-\frac{a_{o}}{8} \sigma^{-2}(x)\left|w_{k_{o}}(x)\right|, \quad x \in \Omega .
\end{gathered}
$$

Proof. From (4.10), (4.11), and hypothesis $\left(h_{1}\right)$, we deduce that

$$
\begin{gathered}
\sigma\left|\sum_{i, j=1}^{n} a_{i j} \frac{\left(\eta_{k}^{s}\right)_{x_{j}}}{\eta_{k}^{s}}\right| \leq c_{4}\left(\eta_{k}\right)_{x}, \\
\sigma^{2}\left|\sum_{i, j=1}^{n} a_{i j} \frac{\left(\eta_{k}^{s}\right)_{x_{i}}\left(\eta_{k}^{s}\right)_{x_{j}}}{\left(\eta_{k}^{s}\right)^{2}}\right|+\sigma^{2}\left|\sum_{i, j=1}^{n} a_{i j} \frac{\left(\eta_{k}^{s}\right)_{x_{i} x_{j}}}{\eta_{k}^{s}}\right| \leq c_{5}\left(\left(\eta_{k}\right)_{x}^{2}+\eta_{k}\left(\eta_{k}\right)_{x x}\right), \\
\sigma^{2}\left|\sum_{i=1}^{n} a_{i} \frac{\left(\eta_{k}^{s}\right)_{x_{i}}}{\eta_{k}^{s}}\right| \leq c_{6}\left(\eta_{k}\right)_{x},
\end{gathered}
$$

where $c_{4}, c_{5} \in \mathbb{R}_{+}$depend on $s, n, v_{0}$ and $c_{6} \in \mathbb{R}_{+}$depends on $s, n, v_{1}$. Observing that $\left(\eta_{k}\right)_{x}=\left(\eta_{k}\right)_{x x}=0$ in $\bar{\Omega}_{k}$, the statement follows now from (4.8), (4.9), $\left(h_{1}\right),\left(h_{2}\right)$, and (4.24).

\section{Main results}

It is well know that there exists a function $\tilde{\alpha} \in C^{\infty}(\Omega) \cap C^{0,1}(\bar{\Omega})$ which is equivalent to $\operatorname{dist}(\cdot, \partial \Omega)$ (see, e.g., [8]). For every positive integer $m$, we define the function

$$
\psi_{m}: x \in \bar{\Omega} \longrightarrow g(m \tilde{\alpha}(x))\left(1-g\left(\frac{|x|}{2 m}\right)\right),
$$

where $g \in C^{\infty}\left(\overline{\mathbb{R}}_{+}\right)$verifies (4.1). It is easy to show that $\psi_{m}$ belongs to $C_{o}^{\infty}(\Omega)$ for every $m \in \mathbb{N}$ and

$$
0 \leq \psi_{m} \leq 1, \quad \operatorname{supp} \psi_{m} \subseteq E_{2 m}, \quad \psi_{\left.m\right|_{\bar{E}_{m}}}=1,
$$

where

$$
E_{m}=\left\{x \in \Omega:|x|<m, \tilde{\alpha}(x)>\frac{1}{m}\right\}
$$


Remark 5.1. It follows from hypothesis $\left(h_{1}\right)$ and from [5, Lemma 4.2] that for any $m \in \mathbb{N}$ the functions $\left(\psi_{m} a_{i j}\right)_{o}$ (obtained as extensions of $\psi_{m} a_{i j}$ to $\mathbb{R}^{n}$ with zero values out of $\Omega$ ) belong to $\operatorname{VMO}\left(\mathbb{R}^{n}\right)$ and

$$
\left[\left(\psi_{m} a_{i j}\right)_{o}\right]_{\mathrm{BMO}\left(R^{n}, t\right)} \leq\left[\psi_{m} a_{i j}\right]_{\mathrm{BMO}(\Omega, t)}
$$

for $t$ small enough.

In the following we denote by $w, b_{i}, b$, and $g$ the functions defined by (4.14), (4.22), respectively, corresponding to $k=k_{o}$, where $k_{o}$ is the positive integer of Lemma 4.2

We can now prove the main result of the paper.

Theorem 5.2. Suppose that conditions $\left(h_{1}\right)$ and $\left(h_{2}\right)$ hold, and let $u$ be a solution of the problem $(P)$. Then there exist an open ball $B \subset \subset \Omega$ and a constant $c_{o} \in \mathbb{R}_{+}$such that

$$
\sup _{\Omega} \sigma^{s}(x) u(x) \leq c_{o}\left(f_{B}\left|\sigma^{s+2} f^{-}\right|^{p} d x\right)^{1 / p},
$$

where $c_{o}$ depends only on $n, p, s, \gamma, v, \nu_{0}, \nu_{1}, v_{2}, a_{o}, \eta\left[\psi_{m} a_{i j}\right](m \in \mathbb{N})$.

Proof. It can be assumed that $\sup _{\Omega} \sigma^{s}(x) u(x)>0$. Thus it follows from (4.14) and (4.18) that there exists $y \in \Omega$ such that $\sup _{\Omega} w(x)=w(y)$; moreover, there exists $\left.R_{o} \in\right] 0$, $\operatorname{dist}(y, \partial \Omega)\left[\right.$ such that $w(x)>0$ for all $x \in B\left(y, R_{o}\right)$.

Let $\lambda, \alpha, \alpha_{o} \in \mathbb{R}_{+}$, with $\alpha_{o}>1$ (that will be chosen late), such that

$$
\lambda \alpha \leq \min \left\{R_{o}, \sigma(y)\right\}, \quad \alpha=\alpha_{o} \sigma(y) .
$$

In the following we denote by $B$ the open ball $B(y, \alpha \lambda)$.

We put

$$
\varphi(x)=1+\lambda^{2}-\frac{|x-y|^{2}}{\alpha^{2}}, \quad x \in \bar{B},
$$

and observe that

$$
\begin{gathered}
1 \leq \varphi(x) \leq 1+\lambda^{2} \leq 2, \quad x \in \bar{B}, \\
\varphi_{x_{i}} \leq \frac{2 \lambda}{\alpha}, \quad \varphi_{x_{i}} \varphi_{x_{j}} \leq \frac{4 \lambda^{2}}{\alpha^{2}}, \quad i, j=1, \ldots, n, \\
\varphi_{x_{i} x_{j}}=0 \quad \text { if } i \neq j, \quad \varphi_{x_{i} x_{j}}=-\frac{2}{\alpha^{2}} \quad \text { if } i=j .
\end{gathered}
$$

Consider now the function $v$ defined by

$$
v(x)=\varphi(x) w(x)-w(y), \quad x \in \bar{B} .
$$

Obviously,

$$
v_{\left.\right|_{\partial \Omega}}=w_{\left.\right|_{\partial \Omega}}-w(y) \leq 0, \quad v(y)=\lambda^{2} w(y) .
$$


It is easy to show that

$$
\begin{aligned}
& L_{o}(\varphi w)-w L_{o} \varphi-2 \sum_{i, j=1}^{n} a_{i j} \varphi_{x_{j}} w_{x_{i}}+\sum_{i=1}^{n} b_{i}(\varphi w)_{x_{i}} \\
& \quad-\sum_{i=1}^{n} b_{i} \varphi_{x_{i}} w+b \varphi w=\varphi\left(L_{o} w+\sum_{i=1}^{n} b_{i} w_{x_{i}}+b w\right) \geq \varphi g \quad \text { in } B .
\end{aligned}
$$

Thus

$$
L_{o}(\varphi w)+\sum_{i=1}^{n} d_{i}(\varphi w)_{x_{i}}+d \varphi w \geq \varphi g+\sum_{i=1}^{n} b_{i} \varphi_{x_{i}} w \quad \text { in } B,
$$

where

$$
\begin{aligned}
& d_{i}=b_{i}-2 \sum_{j=1}^{n} a_{i j} \frac{\varphi_{x_{j}}}{\varphi}, \quad i=1, \ldots, n, \\
& d=b+2 \sum_{i, j=1}^{n} a_{i j} \frac{\varphi_{x_{i}} \varphi_{x_{j}}}{\varphi^{2}}-\sum_{i, j=1}^{n} a_{i j} \frac{\varphi_{x_{i} x_{j}}}{\varphi} .
\end{aligned}
$$

Therefore we obtain from (5.14) that

$$
L_{o} v+\sum_{i=1}^{n} d_{i} v_{x_{i}}+d v \geq h,
$$

where

$$
h=\varphi g+w \sum_{i=1}^{n} b_{i} \varphi_{x_{i}}-d w(y) .
$$

Clearly, (2.9), (5.6), and (5.9) yield that

$$
\left|\varphi_{x_{i}}\right| \leq 2 \gamma \frac{\sigma}{\alpha_{o}^{2} \sigma^{2}(y)} \quad \text { in } B
$$

and hence it follows from Lemma 4.2 that

$$
\begin{aligned}
h & \geq \varphi \eta_{k_{o}}^{s} f-\frac{a_{o}}{8} \sigma^{-2} \varphi w(y)-2 \gamma w(y)\left(\nu_{1}+\frac{a_{o}}{2}\right) \frac{1}{\alpha_{o}^{2}} \sigma^{-2}(y)-d w(y) \\
& \geq \varphi \eta_{k_{o}}^{s} f+\left[-d-\left(\frac{a_{o}}{4} \gamma^{2}+2 \frac{\gamma \nu_{1}}{\alpha_{o}^{2}}+\frac{\gamma a_{o}}{\alpha_{o}^{2}}\right) \sigma^{-2}(y)\right] w(y) .
\end{aligned}
$$

The constant $\alpha_{o}$ can be chosen in such a way that $d<-d_{o} \sigma^{-2}(y)$ in $B$, where

$$
d_{o}=\frac{a_{o}}{4} \gamma^{2}+2 \frac{\gamma \nu_{1}}{\alpha_{o}^{2}}+\frac{\gamma a_{o}}{\alpha_{o}^{2}}
$$


12 Bounds for elliptic operators in weighted spaces

In fact, by Lemma 4.2, (5.9) and (5.10), we have

$$
\begin{aligned}
d+d_{o} \sigma^{-2}(y) & =b+2 \sum_{i, j=1}^{n} a_{i j} \frac{\varphi_{x_{i}} \varphi_{x_{j}}}{\varphi^{2}}-\sum_{i, j=1}^{n} a_{i j} \frac{\varphi_{x_{i} x_{j}}}{\varphi}+d_{o} \sigma^{-2}(y) \\
& \leq-\frac{a_{o}}{2} \sigma^{-2}+8 v_{o} \frac{\lambda^{2}}{\alpha^{2}}+2 v_{o} \frac{1}{\alpha^{2}}+d_{o} \sigma^{-2}(y) \\
& \leq\left[-\gamma^{2} \frac{a_{o}}{4}+\left(10 v_{o}+2 \gamma \nu_{1}+\gamma a_{o}\right) \frac{1}{\alpha_{o}^{2}}\right] \sigma^{-2}(y),
\end{aligned}
$$

and hence, fixed $\alpha_{o}$ such that

$$
\frac{1}{\alpha_{o}^{2}} \leq \frac{\gamma^{2} a_{o}}{4\left(10 v_{o}+2 \gamma \nu_{1}+\gamma a_{o}\right)}
$$

it follows that

$$
d<-d_{o} \sigma^{-2}(y) \text { in } B
$$

By (5.11), (5.12), and (5.15)-(5.17), we deduce that the problem

$$
\begin{gathered}
v \in W^{2, p}(B), \\
L_{o} v+\sum_{i=1}^{n} d_{i} v_{x_{i}}+d v \geq \varphi \eta_{k_{o}}^{s} f, \quad f \in L^{p}(B), \\
v_{\left.\right|_{\partial B}} \leq 0
\end{gathered}
$$

satisfies the hypotheses of Lemma 3.1. Therefore, it follows from (5.6), (4.15), and (4.16) that there exists a constant $c_{1} \in \mathbb{R}_{+}$, depending on $n, p, s, \gamma, v, v_{0}, v_{1}, v_{2},\left[p\left(a_{i j_{\left.\right|_{B}}}\right)\right]_{\mathrm{BMO}\left(R^{n}, \cdot\right)}$, such that

$$
v(x) \leq c_{1}(\lambda \alpha)^{2-n / p}\left\|\left(\varphi \eta_{k_{o}}^{s} f\right)^{-}\right\|_{L^{p}(B)} \quad \forall x \in B .
$$

So it follows from (5.8) and from (5.26) with $x=y$ that

$$
\lambda^{2} w(y) \leq c_{1}(\lambda \alpha)^{2-n / p}\left\|\left(\varphi \eta_{k_{o}}^{s} f\right)^{-}\right\|_{L^{p}(B)} \leq 2 c_{1}(\lambda \alpha)^{2-n / p}\left\|\eta_{k_{o}}^{s} f^{-}\right\|_{L^{p}(B)} .
$$

Thus by (5.6) and (5.27) we have

$$
w(y) \leq c_{2}(\lambda \alpha)^{-n / p} \alpha_{o}^{2} \sigma^{2}(y)\left\|\eta_{k_{o}}^{s} f^{-}\right\|_{L^{p}(B)} \leq c_{3}(\lambda \alpha)^{-n / p} \alpha_{o}^{2}\left\|\sigma^{2} \eta_{k_{o}}^{s} f^{-}\right\|_{L^{p}(B)},
$$

where $c_{2}, c_{3} \in \mathbb{R}_{+}$depend on the same parameters as $c_{1}$. Finally from (4.6), (4.7), (4.14), and (5.28) we obtain

$$
\sup _{\Omega} \sigma^{s} u \leq c_{4}(\lambda \alpha)^{-n / p}\left(\int_{B}\left|\sigma^{2+s} f^{-}\right|^{p} d x\right)^{1 / p} \leq c_{5}\left(f_{B}\left|\sigma^{s+2} f^{-}\right| d x\right)^{1 / p},
$$


where $c_{4}, c_{5} \in \mathbb{R}_{+}$depend on the same parameters as $c_{1}$ and on $a_{o}$. Then, if we choose

$$
p\left(a_{i j_{B}}\right)=\left(\psi_{m_{1}} a_{i j}\right)_{o},
$$

where $m_{1}$ is a positive integer such that $\psi_{\left.m_{1}\right|_{B}}=1$, (5.5) follows from (5.29), (5.30), and from Remark 5.1.

Corollary 5.3. Suppose that conditions $\left(h_{1}\right)$ and $\left(h_{2}\right)$ hold, and let $u$ be a solution of the problem

$$
\begin{gathered}
L u=f, \quad \sigma^{s+2} f \in L^{\infty}(\Omega), \quad u \in W_{\text {loc }}^{2, p}(\Omega), \\
\limsup _{x \rightarrow x_{o}} \sigma^{s}(x) u(x)=0 \quad \forall x_{o} \in \partial \Omega, \\
\limsup _{|x| \rightarrow+\infty} \sigma^{s}(x) u(x)=0 \quad \text { if } \Omega \text { is unbounded. }
\end{gathered}
$$

Then

$$
\sup _{\Omega} \sigma^{s}|u| \leq c_{o}\left\|\sigma^{s+2} f\right\|_{L^{\infty}(\Omega)}
$$

where $c_{o} \in \mathbb{R}_{+}$is the constant of the statement of Theorem 5.2.

Proof. The result can be obtained applying Theorem 5.2 to the functions $u$ and $-u$.

The following uniqueness result is an obvious consequence of Corollary 5.3.

Corollary 5.4. If the hypotheses $\left(h_{1}\right)$ and $\left(h_{2}\right)$ hold, then the problem

$$
\begin{gathered}
L u=0, \quad u \in W_{\text {loc }}^{2, p}(\Omega), \\
\limsup _{x \rightarrow x_{o}} \sigma^{s}(x) u(x)=0 \quad \forall x_{o} \in \partial \Omega, \\
\limsup _{\sigma^{s}(x) u(x)=0} \quad \text { if } \Omega \text { is unbounded }
\end{gathered}
$$

has only the zero solution.

\section{References}

[1] L. Caso, P. Cavaliere, and M. Transirico, On the maximum principle for elliptic operators, Mathematical Inequalities \& Applications 7 (2004), no. 3, 405-418.

[2] L. Caso and M. Transirico, Some remarks on a class of weight functions, Commentationes Mathematicae Universitatis Carolinae 37 (1996), no. 3, 469-477.

[3] F. Chiarenza, F. Frasca, and P. Longo, $W^{2, p}$-solvability of the Dirichlet problem for nondivergence elliptic equations with VMO coefficients, Transactions of the American Mathematical Society 336 (1993), no. 2, 841-853.

[4] C. Pucci, Limitazioni per soluzioni di equazioni ellittiche, Annali di Matematica Pura ed Applicata 74 (1996), 15-30.

[5] M. Transirico, M. Troisi, and A. Vitolo, BMO spaces on domains of $\mathbb{R}^{n}$, Ricerche di Matematica 45 (1996), no. 2, 355-378.

[6] M. Troisi, Su una classe di funzoni peso, Rendiconti. Accademia Nazionale delle Scienze detta dei XL. Serie V. Memorie di Matematica 10 (1986), no. 1, 141-152. 
14 Bounds for elliptic operators in weighted spaces

[7] C. Vitanza, A new contribution to the $W^{2, p}$ regularity for a class of elliptic second order equations with discontinuous coefficients, Le Matematiche 48 (1993), no. 2, 287-296.

[8] W. P. Ziemer, Weakly Differentiable Functions, Graduate Texts in Mathematics, vol. 120, Springer, New York, 1989.

Loredana Caso: Dipartimento di Matematica e Informatica, Facoltà di Scienze MM.FF.NN., Università di Salerno, Via Ponte don Melillo, 84084 Fisciano (SA), Italy

E-mail address: lorcaso@unisa.it 